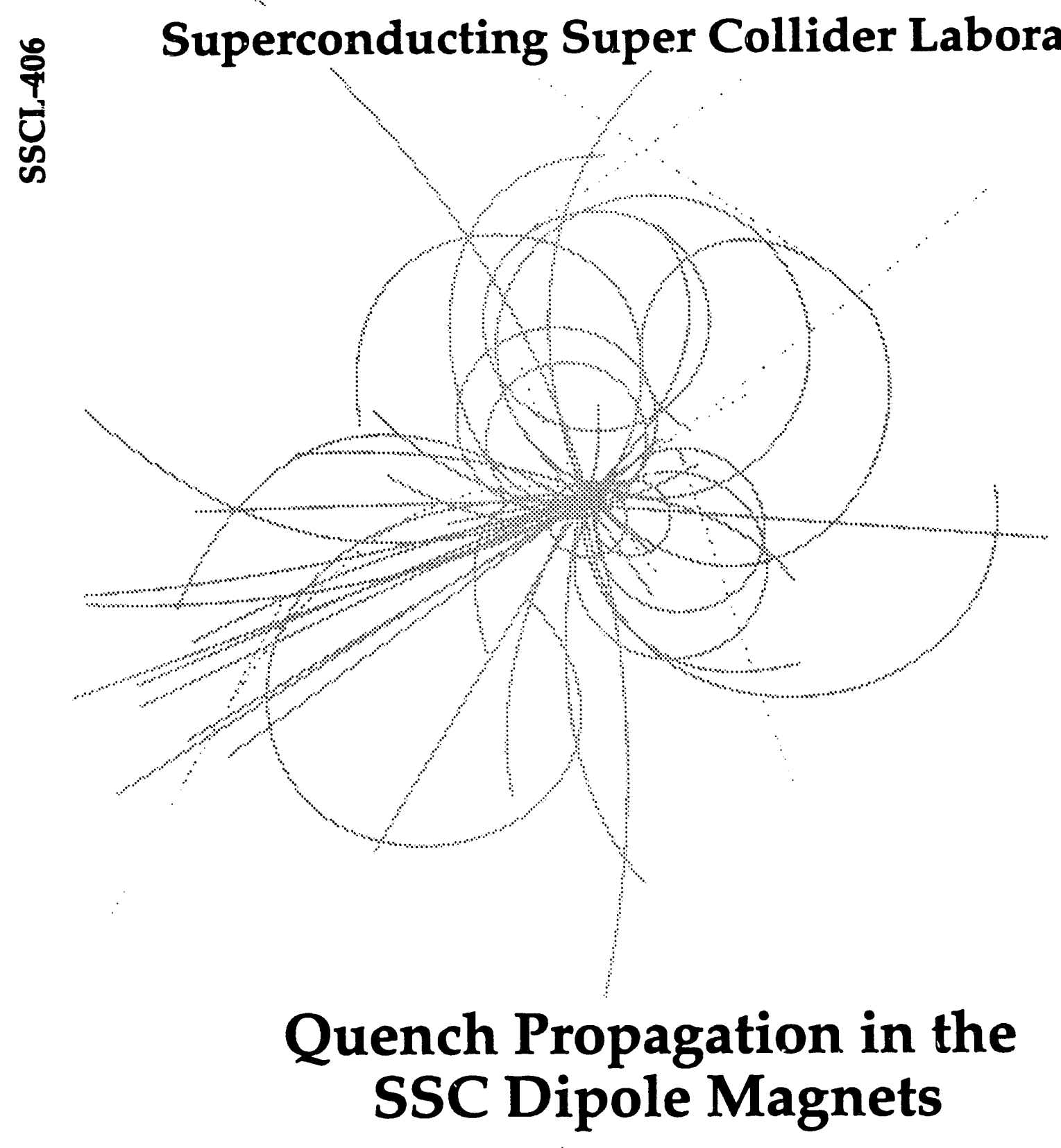

G. López and G. Snitchler

September 1990 


\title{
Quench Propagation in the SSC Dipole Magnets*
}

\author{
G. López and G. Snitchler \\ Accelerator and Magnet Divisions \\ Superconducting Super Collider Laboratory ${ }^{\dagger}$ \\ 2550 Beckleymeade Ave. \\ Dallas, TX 75237
}

September 1990

\footnotetext{
"Submitted to the Applied Superconductivity Conference, Snowmass, Colorado, September 24-28, 1990.

tOperated by the Universities Research Association, Inc., for the U.S. Department of Energy under Contract No. DE-AC02-89ER40486.
} 


\title{
QUENCH PROPAGATION DN THE SSC DIPOLE MAGNETS
}

\author{
G. Lopez and G. Snitchler \\ Superconducting Super C'sllider Laboratory \\ 2550 Beckleymeade \\ Dallas, TX 75237
}

\begin{abstract}
Abssends
The effects of quench propagation are modeled in $40 \mathrm{~mm}$ and $50 \mathrm{~mm}$ diameter collider dipole magnet designs. A comparative study of the cold diode (passive) and quench heater (active) prolection schemes will be presented. The SSCQ modeling program accuraiely simulares the axial quench velocity and uses phenomenological time delays for num-10-aum oransverse propagation. The arial quench velocity is field dependent and consequendy, each conducior's quench profile is racked separately. No symmetry constraints are employed and the distribution of the lemperatures along the conductor differs from the adiabatic approximation. A single magnet has a wide margin of self protection which suggests that passive protection schemes must be considered.
\end{abstract}

\section{Introduclion}

In the current configuration, the apenure size of the long SSC collider dipole magnets (CDM) has been changed from $40 \mathrm{~mm}$ to $50 \mathrm{~mm}$. While the test results in long $40 \mathrm{~mm}$ CDM rest magnets reveal that the observed fast quench velocities provide excellent quench protection, the remaining concern is that if the SOmm magnets propagate significantly slower than the $40 \mathrm{~mm}$ design there could be difficulty in designing a protection scheme. Currently, there is no explanation for the unusually fast quench velocities in the long $40 \mathrm{~mm}$ CDM magness.

The unexplained quench propagation velocities only oceur in long $40 \mathrm{~mm}$ SSC dipole magnets which are $17 \mathrm{~m}$ in kngh. The quench velocity governs the resistance development in the coil which in rum govems the current decay. A rapid curreat decay inplies a lower maximum temperanure and is usually represented in terrns of the integral of $I^{2}$ over all time after initiacion of the guench and is refered to as MUTS. The number of MIITS must be limited to protect the miterials and the integrity of the cold mass. In the current lone $40 \mathrm{~mm}$ SSC dipole magnets, the plateau quencb normal zone appears to propagate with extremely fast velocities, nesr 1400n's on the inner pole-tum cable. In contrest, the plateau quench velocities in stion SSC dipole magnets, $1.80 \mathrm{~m}$ in length, are oear 80 meters/second' which is the theronal conductive propagation limit ${ }^{2}$ Recent experimental evidence suggests that the quench velocities accelerate from $100 \mathrm{~m} / \mathrm{s}$ to $150 \mathrm{~m} / \mathrm{s}$ if the quench propagates down the full length of the magnet. 3

A preliminary model of quench events in lons $40 \mathrm{~mm}$ dipole magnets is based on an empirical approach. The adiabatic quench velocities are normally expressed in terms of current density fraction-of-shon-sample, q. The adiabatic quench velocity expression can be modified to fit experimental data from $40 \mathrm{~mm}$ long magnet test data. Test magnet tum-10tum asmuthal propagation test delays and inner-outer coil times delays are employed to predict maximum temperanures in the coil.
There is a pressing need to predict the performance of the impending long $50 \mathrm{~mm}$ dipole magnets. The first long protolype should be completed in the fall of 1991 . The long $40 \mathrm{~mm}$ magnet model has been extended to the SOmoo design assuming the longitudinal quench velocity and all aximutha and inner. outer coil time delays have the same dependence of $q$. This model is used to predict active and passive protection schemes and their effectiveness 10 moderate maximum temperatures developed in a quench event.

\section{Quench Model}

The preliminary quench model for the $40 \mathrm{~mm}$ designs has been completed. Since the limit of thermal conduction quench velocities in SSC dipole magnets is approximately 80 meters/second, an empirical technique is employed 10 approximate the faster quench velocities and the temperanure profule in SCC long dipole magnets. The standard quench velocity expression is

$$
\nu_{08}=\frac{J_{9}}{\delta c_{8}} \sqrt{\frac{k p}{\left(\theta_{8}-\theta_{0}\right)}}
$$

where $\mathrm{J}_{0}$ is the current density, $\mathbf{8 C _ { g }}$ is the density imes the heat capacity, $k$ is the copper thermal conductivity, $p$ is resistivity. $\theta_{0}$ is the batch temperature, and $\theta_{8}$ is the temperature that the heat generation step function turns on.4 $\theta_{s}$ is usually approximated by $\theta_{s}=\left(\theta_{g}+\theta_{c}\right) / 2$ where $\theta_{g}$ is the current sharing temperanure. $\theta_{\mathrm{c}}$ is the crivical temperanre. This expression is modified such that the quench velocity matches near shor sample quenches.

$$
\theta_{s}=\theta_{g}+\delta \theta(q)
$$

where $\theta_{s}$ is the adjusted heal generation step function which is then inserned in equation 1 to produce $V_{a g}$, the adjusted quench velocity. The term $80(q)$ is a fit parameter such that

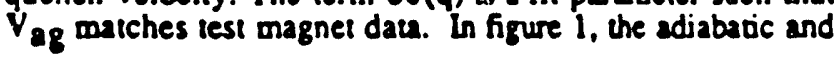
adjusted quench velocities are plotied against test magnet quench date

The quench temperature profile of each conductor is tracked, and temperature and B.field dependent material properties determine the quench velocities, based on $V_{a g}$. Maximum temperanures are presented in table $l$ and are a factor of 2 higher than experimental data. This model differs from test conditions in that all turn-to-tum time delays are assumed to be maintained and all conductors 80 bormal sequentially. Also. 
this single model did not contain a heater firing representation and that the inner coil copper-10-superconductor ratio is $\lambda=1.3$. The only hot-spot experimental data available was on a magnet with $\lambda=1.6$ and the heaters were fired with no ime delay. An additional model using $\lambda=1.6$ produced a hot spot temperalure approximately $100 \mathrm{~K}$ lower than the model results presented in the table. Other test results ?':z,jest this test data had a reduced peak temperature, approximaiely SOK lower, due to the heater firing.s There are several adjustments which can be made to refine this preliminary model. Also, there is a need for more hot-spot temperature measurements based on more recent magnet designs which are closer to the $40 \mathrm{~mm}$ design baseline.

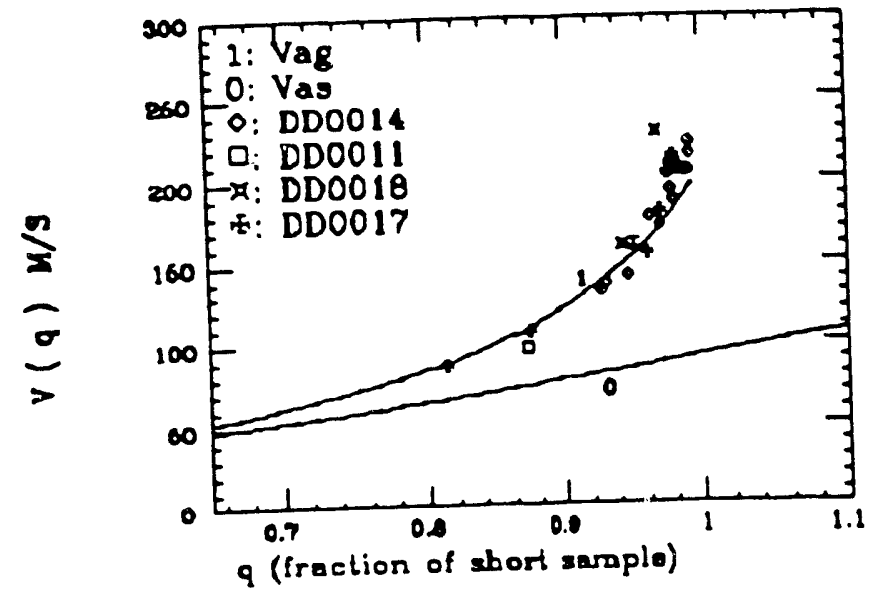

Fig. 1- Adjusted vs. adiabatic quench velocities

This rechnique may yield uncerainties in predicting the behavior of a new design because the quench velocities are not well understood; however, it can suggest relative design performance. Unfortunately, this technique is empirical and does not represent any physical process. Comparative models can only be made by scaling the nomalously accelerated propagation as a function of $\mathbf{q}$.

\begin{tabular}{|l|r|r|r|}
\hline Table 1 & $\begin{array}{r}40 \mathrm{~mm} \\
\text { baseline }\end{array}$ & DDO017† & $\begin{array}{r}50 \mathrm{~mm} \\
\text { baseline }\end{array}$ \\
\hline Cu:S.C. ratio inner & 1.3 & 1.6 & 1.5 \\
Cu:S.C. ratio outer & 1.8 & 1.8 & 1.8 \\
inner cable area & 11.79 & & 15.37 \\
outer cable area & 9.89 & & 11.87 \\
Stored Energy: & $1.2 \mathrm{MJ}$ & $1.2 \mathrm{MJ}$ & $1.57 \mathrm{MJ}$ \\
\hline & Model & Expt. & Model \\
\hline Max temperature & 390 & 157 & 310 \\
MIITS & 9.64 & 7.39 & 19.5 \\
\hline †40mm test magnet, Ref́erence 5. \\
\hline
\end{tabular}

This model predicts that the $50 \mathrm{~mm}$ design will experience peak temperatures half that of the $40 \mathrm{~mm}$ design. This approximately agrees with simple adiabatic "MIITS" approximations using empirical fits to experimental data which concludes that the $50 \mathrm{~mm}$ design can sustain 20 MUITS before reaching $800 \mathrm{~K}$ vs. $12 \mathrm{MUITS}$ in the $40 \mathrm{~mm}$ design. 6 The major prolisction difference in these designs is the $3020 \%$ increase in cross-sectional area in the inner/outer conductor as seen in Table 1. Using an empirical model, a relative comparison of
$40 \mathrm{~mm}$ vs. $50 \mathrm{~mm}$ designs suggests that the $50 \mathrm{~mm}$ design will be easier to protect and obtain lower maximum iemperarures.

\section{Aclive Prolection Models}

This model has been adapted 10 model magnet protection in active and passive schemes. The active protection system may include up to five long SSC dipole magnets and a quadrupole magnet which are isolated from the sector power supply by a warm diode. The passive protection system would isolate an individual magnet with a cold diode under the assumption that a single magnet is "self protected" and no heaters are needed to protect it. In both schemes, the non-quenching sector magnets are ramped down in approximately 30 seconds.

If an active protection system is employed to protoc: a halfcell of magnets, the stored energy of some or all of the 6 magnets must be distributed between all magnets in the halfcell. The current protection baseline states that the half-cell will be divided into two active protection sub-groups each separated from the sector by a warm diode. Each magnet has active two outer-pole-tum strip heaters. Each strip heater contains twentyfour heater pads which forces ten cables into a normal state down the length of the magnet. In the event of a quench, the warm diode will isolate the 3 magneas.

A modification of the model described previously was used to model a quench event in a series circuit of magnets. A string of magnets in series was modeled with one heater firing on both sides of the magnet to emulate the active protection scheme. The heater time delays represent both voltage detection and systemic ame delays in firing the heaters. The heater trigger specification is 0.5 volts actoss the magnet In figure 2 , the results of several total time delays are presented. These results demonstrate that there is a clear maximum acceptable time delay before firing the heaters to protect the half-cell This one heater model provides a pessimistic view of quench development in the outer coil. The baseline configuration has 24 heaters in series on each side of the magnet and improved models based on 24 heaters would yield more optimistic results.

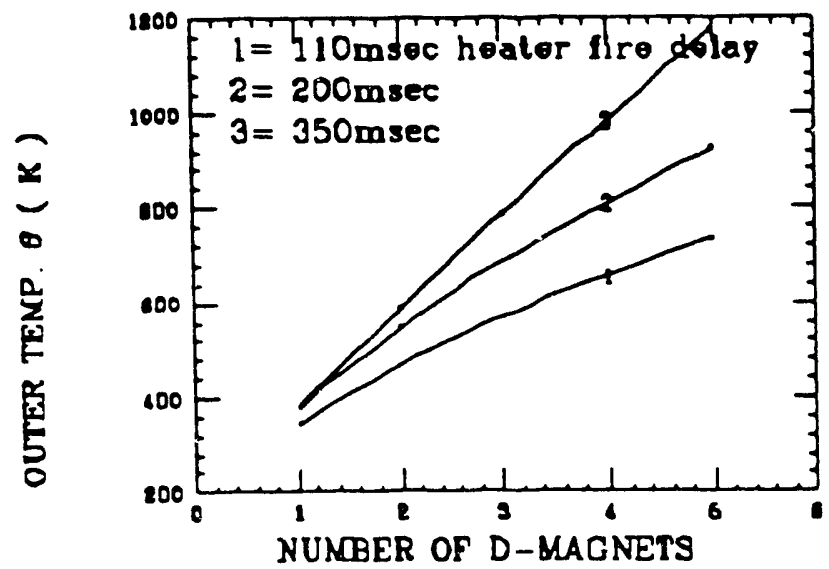

Fig. 2 - Hot Spot Temperatures

The second technique involves using the resistance development from magnet test data to predict string performance. This is a simple and more accurate model which 
is based on the curtent vs. time data from test magnets. A resistance vs. time profile is calculated and it reproduces the inductance-resistance (LR) circuit current vs. time profile. By using a time dependent model of the resistivity development during spontaneous and heater induced quenches, one can develop a new current profile for a series of magnets and predict MIITS for the spontaneously quenching magnet assuming the inductance is known. This is not completely accurate because resistivity would develop faster in the case of higher time delays with more stored energy. The case chosen was developed from curtent vs. time tesi data from a near shor-sample quench for the test magnet DD0027. The only safery-heater induced quench test data occurring near shor sample was a 6600 amp quench found in DD0019 and this current vs. time profile was almost identical to the resistivity development in the spontaneous 6800 amp quench in DD0027.

Using the resistance profile from the previously mentioned spontaneous DD0027 quench for spontaneous, $R_{s}(t)$, and heater induced quenches, $R_{h}(t), a L R$ circuit was modeled based on the inductance of $N$ magnets where $(N-1)$ magnets had a firing time delay modeled by offsetting the resistance development for (N-1) magnets. The current profile is

$$
I=I_{0} \operatorname{Exp}\left[-t\left\{R_{s}(t)+(N \cdot 1) R_{b}(t+d)\right] /(N L)\right]
$$

where $d$ is the time delay and $I_{0}$ is the initial current. In figure 3. the MIITS performance is ploned in relationship to heater firing delay times and the number of dipole magnets in the string. As the firing time increases, the spontaneously quenching magnet coninues 10 heat-up due to the stored energy of all the magnets in series. 8 MUITS corresponds to a hot-spod temperature of $200 \mathrm{~K}$ and 12 MUTTS corresponds to $800 \mathrm{~K}$ for a $40 \mathrm{~mm}$ inner coil. The $800 \mathrm{~K}$ number has nor been observed, it is only derived from the adiabatic approximation.

Qualitatively, the nwo models agree that active protection is feasible. The following results suggest that if a quench can be detected and the heaters fire in $40-100 \mathrm{msec}$, the half cell would be properly prorected

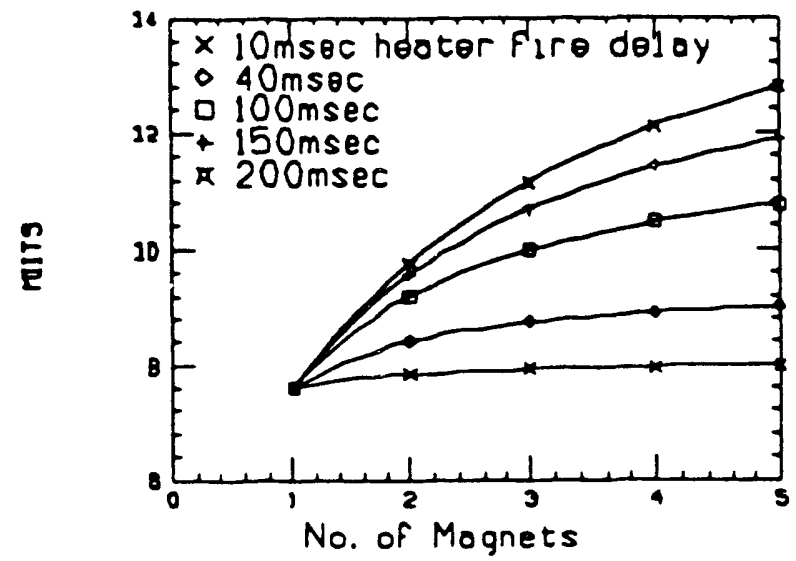

Fig. 3 - Active Protection $40 \mathrm{~mm}$ MIITS

\section{Conclusions}

Empirical passive protection models predict that the $50 \mathrm{~mm}$ magnets are more protected than the current $40 \mathrm{~mm}$ test magnets. The $50 \mathrm{~mm}$ design is more protected because the reduction of the current density in copper slows down the resistivity development and power dissipation; however, lower current densities also slow down the quench velocities and these models may not accurately predict quench velocities in untested magnet designs.

The resistive development active protection model predicts with proper quench detection that an active protection system will also provide adequate magnet protection. The models strongly depend on test data from the $40 \mathrm{~mm}$ design. The heat development and quench event will develop slower in the $50 \mathrm{~mm}$ design facilitating more margin for heater reaction time; however, higher Jc margin slows down healer reaction. This also implies that fast acting heaters could further reduce peak temperatures. Also, active protection may provide the best protection for outer coil quenches which tend to have slower quench velocities.

The planned long sample tests and more complete models will yield more information for $50 \mathrm{~mm}$ long magnet half-cell protection. Hopefully, these tests and models will be completed in time to impact the long $50 \mathrm{~mm}$ dipole magnet program. More complete test data and corresponding models must be developed to accurately describe the near sonic velocities in the long SSC dipoles. The current paradigm includes a helium hydraulic quench event either in the annular space or inside the cable insulation. Long sample tests and more complete models are under development

\section{Beferences}

1 A. Devred and et. al.. unpublished test data on long and shon $40 \mathrm{~mm}$ SSC dipole magnets.

2 G. Lopez, SSC.266 (1990).

32 . Wolf, unpublished data.

$4 M$. Wilson. Superconducting Magnets, Oxford Press. 1983.

5 A. Devred, Investigation of the Hot-Spot

Temperature". SSC MDTA-133 (May 1989).

6 A. Devred. "Safely of $5-\mathrm{cm}$ Apenure Collider

Dipoles". SSC MDTA (May 1990). 

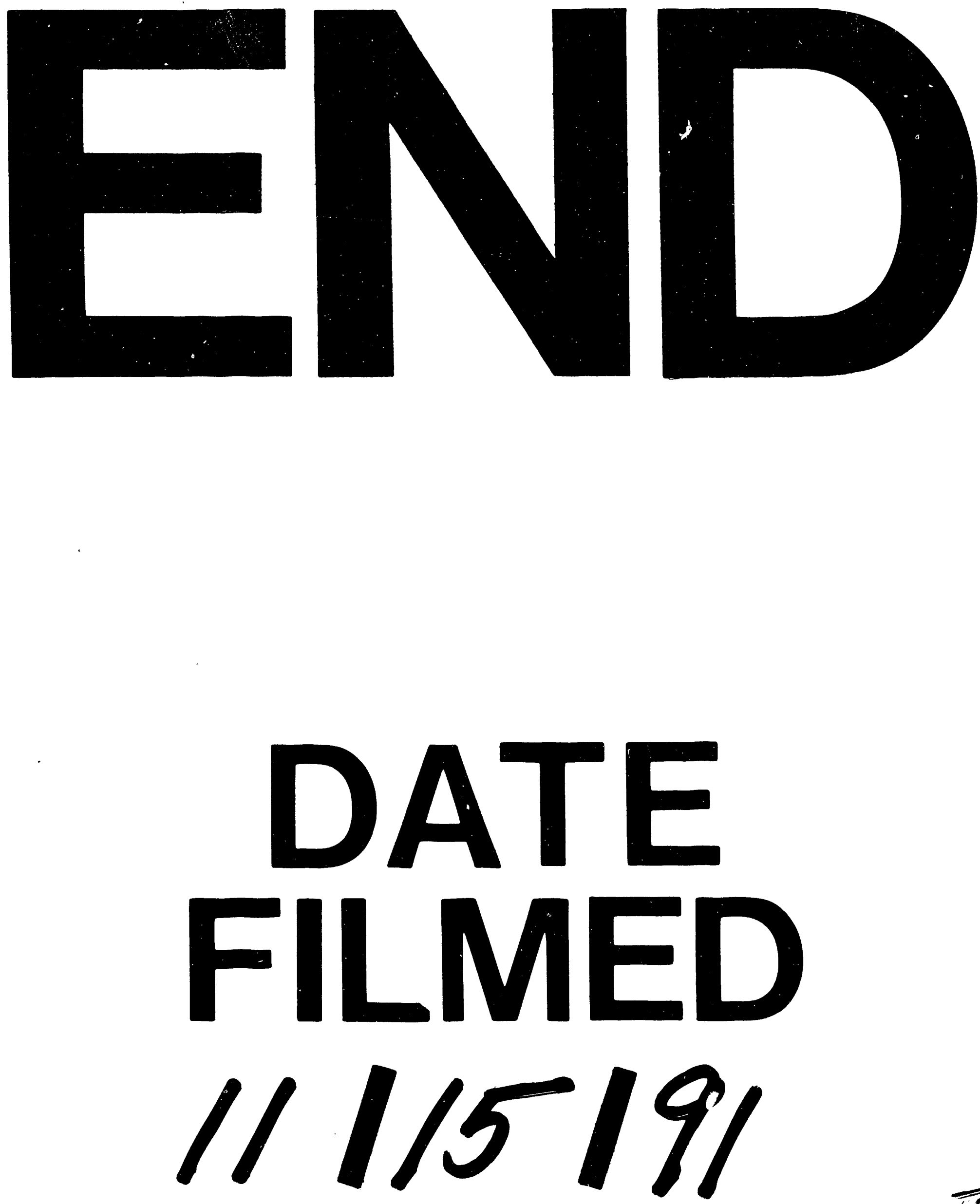
